\title{
Fabrication of three-dimensional SU-8 microchannels by proton beam writing for microfluidics applications: Fluid flow characterisation
}

\author{
S. Al-Shehri*, V. Palitsin, R.P. Webb, G.W. Grime \\ University of Surrey Ion Beam Centre, Advanced Technology Institute, Guildford GU2 7XH, United Kingdom
}

\section{A R T I C L E I N F O}

\section{Article history:}

Received 11 July 2014

Received in revised form 28 November 2014

Accepted 3 December 2014

Available online 30 December 2014

\section{Keywords:}

Proton beam writing

Microfabrication

Microfluidics

Fluid characterisation

Computational fluid dynamics (CFD)

\begin{abstract}
A B S T R A C T
The proton beam writing (PBW) technique was used to fabricate microfluidic structures in SU-8 resist. A network of the buried channels was fabricated as part of a project to develop functional microfluidic device for neuronal studies and self-powered microfluidics. Protons with energies between $2.5 \mathrm{MeV}$ and $0.75 \mathrm{MeV}$ were used to fabricate the buried channels with a minimum feature size of around $1 \mu \mathrm{m}$ and depths of $40-55 \mu \mathrm{m}$. Roughness of channels sidewalls was around $2.5 \mathrm{~nm}$ rms. Exposure regime and examples of functional networks fabricated using PBW are described. COMSOL Multiphysics ${ }^{\circledR}$ software was used to model the flow characteristics of fluid in the SU-8 microchannels structured by PBW. The results obtained using PBW are compared with the structures fabricated by UV-lithography.
\end{abstract}

(C) 2014 Elsevier B.V. All rights reserved.

\section{Introduction}

There is a growing interest in use of microfluidics in biochemistry, medical research, tissue engineering and other applications. It was shown that microfluidic-based systems have a great potential in application for single-cell analysis $[1,2]$ and instant analyses or so called point-of-care (POC) diagnostics [3-5]. In this respect, microfluidics systems provide conditions that are comparable to in vivo conditions [1]. However, to recreate physiologically viable patterns of fluid velocities, microfluidic systems require precise control of fluid flow. Microchannel geometry and surface texture are considered as the crucial factors in determining fluid flow through microchannels. These factors mainly depend on microfabrication techniques and fabrication processes of the microchannels [2-4].

The rapid growth of microfluidic-based single-cell analysis techniques requires a reliable and flexible microfabrication method, which allows producing of micro-features that meet different needs. Fast evaporation of fluid (e.g., biological media, chemical reagents) in the microchannels may cause premature cell death especially in long-term culture assays [5]. To reduce the risk of culture medium evaporation, encapsulation of microchannels is considered an essential requirement. A microfluidic device with a high aspect ratio (HAR) provides a suitable environment for cell

\footnotetext{
* Corresponding author.

E-mail address: s.al-shehri@surrey.ac.uk (S. Al-Shehri).
}

growth, as claimed by Hung et al. [6]. Therefore, production of three-dimensional high aspect ratio microchannels with straight and smooth walls is in high demand.

In contrast to the current microfabrication technologies [7-10], proton beam writing (PBW) is a direct writing technique that allows its use in fabrication three-dimensional microstructures with vertical walls under full control over aspect ratio and surface texture $[7,11,12]$. In comparison to the mask-based techniques [13], PBW is a more flexible and quicker microfabrication process that produces defect-free structures [14].

To date, PBW has successfully been used in the fabrication of microfluidics devices $[11,12,15]$. However, only a small number of studies have used this technique to produce 3D microfluidicbased devices adapted for the single cell analysis [16,17].

In this study, we present the development of Neuron Cell Arrays microfluidics (NCAs) and a microfluidic capillary micro-pump (CMP). The former is to be used for studying the electrical activity patterns in an artificial neural network while the latter is an example of a self-powered microfluidics device. Both microfluidics devices were fabricated in SU-8 photoresist using PBW technique. SU-8 photoresist has excellent mechanical and biocompatibility properties that allow its use in fabrication of the HAR microfluidics for cell study. We developed and successfully deployed a beam scanning algorithm to enhance quality of the fabricated microstructures. In addition, simple numerical simulation models using COMSOL multiphysics were used to characterise the fluid flow pattern in NCAs and CMP microfluidics. 


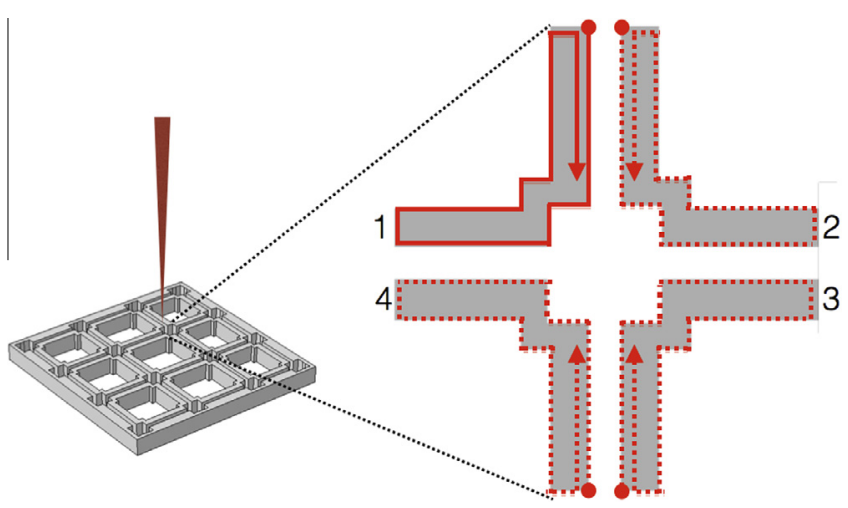

Fig. 1. Schematic diagram illustrates operation of the Edge Following Algorithm (EFA). The red line shows the way of sweeping the beam over SU-8 photoresist to fabricate NCAs structure. (For interpretation of the references to color in this figure legend, the reader is referred to the web version of this article.)

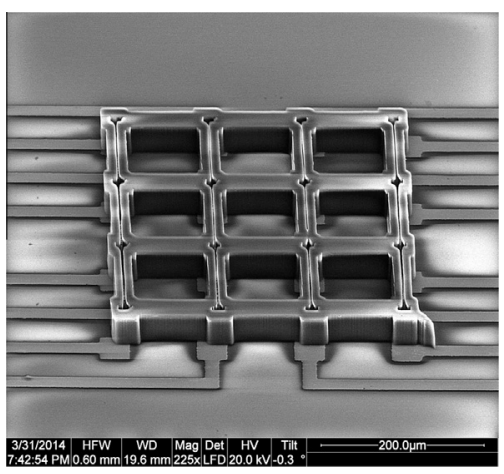

Fig. 2. SEM image of the NCAs structure written in SU-8 by PBW with microchannels of $5 \mu \mathrm{m}$ width aligned over DEP electrodes.

\section{Experimental methods}

\subsection{Three-dimensional microchannels by PBW}

The microfluidics NCAs and CMP were fabricated by using PBW technology at Surrey Ion Beam Centre, University of Surrey. The enclosed channels were structured in $40 \mu \mathrm{m}$ thick SU-8 3000 photoresist (MicroChem Corp.) using proton beams with energies of $2.5 \mathrm{MeV}$ and $0.75 \mathrm{MeV}$ according to the SRIM [18] and following earlier experiments [19]. SU-8 3000 photoresist was chosen as it is better suited to form the thick polymeric films on glass substrates and it exhibits better adhesion and reduced coating stress than commonly used SU-8 2000 photoresist [20]. The NCAs and CMP structures were irradiated with doses ranging from 20 to $30 \mathrm{nC} /$ $\mathrm{mm}^{2}$. The beam current and irradiation doses were determined using RBS detector.

\subsection{Scanning strategy}

Scanning of the proton beam was performed using a magnetic scanning system (OM-25, Oxford Microbeams). We have observed that abrupt changes in the beam scanning directions in some parts of the NCAs outline (e.g., microcompartments edges) generate inductive transients in the scanning system. This appears as some geometrical artefacts in the developed microstructures due to over- or under-shoot of the beam in these areas. Scanning with slower speed can reduce extend of the distortions but will require a longer exposure time. To keep an exposure time to the possible minimum and to reduce the magnetic scanning hysteresis issues the beam should be steered in a parallel path to the channels' outline.

In this study we have assessed two scanning methods, the Ionscan code [21] based on the "turtle" algorithm and the Edge Followed Algorithm (EFA), [22,23] developed in our group. The Ionscan capabilities are limited to an outlining of the basic shapes
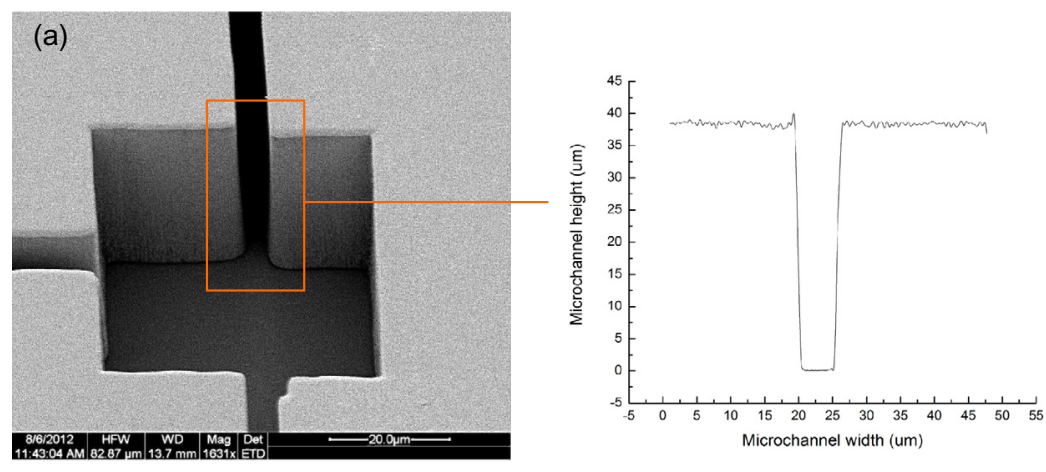

(b)
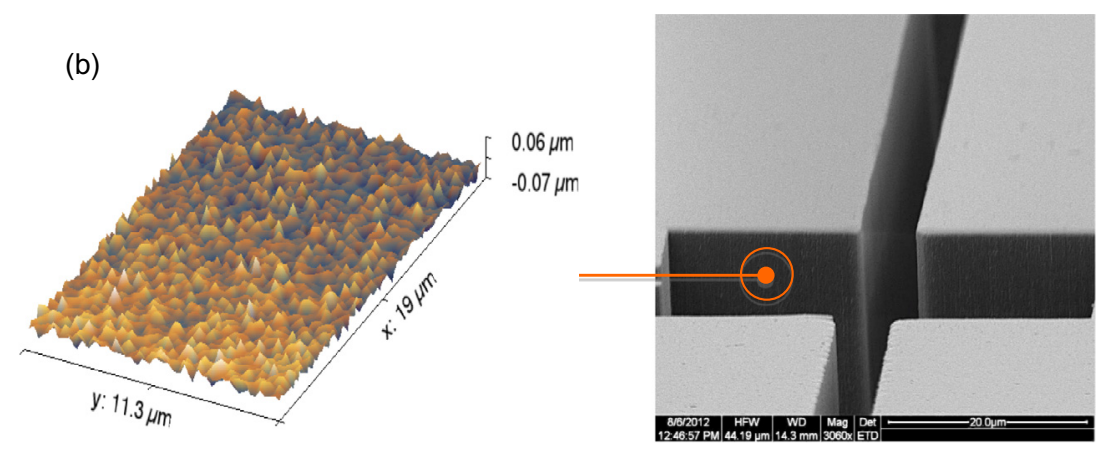

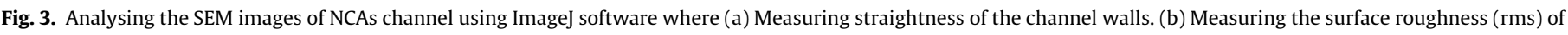
the channel sidewalls. 


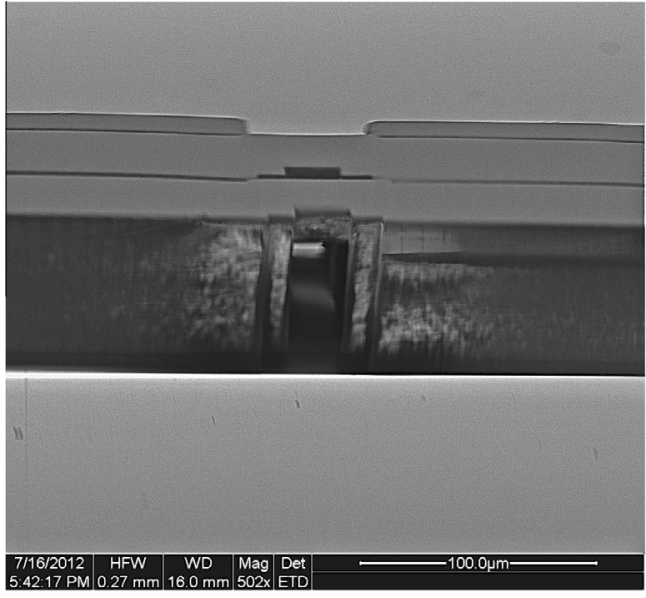

Fig. 4. Tilted SEM image of the buried channels in the NCAs structure fabricated in SU-8 using different proton beam energies, $2.5 \mathrm{MeV}$ and $0.75 \mathrm{MeV}$ respectively.

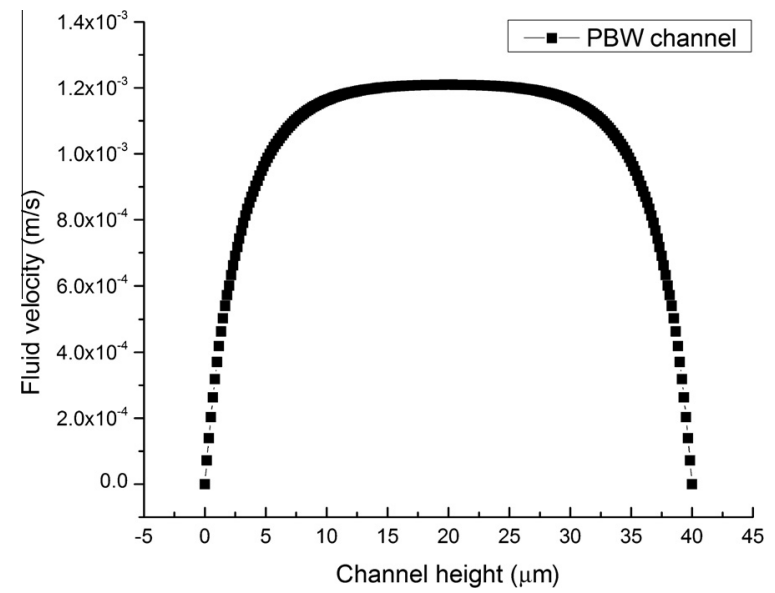

Fig. 5. Velocity profile of a fluid flow modelled numerically in a microchannel fabricated by PBW using COMSOL Multiphysics ${ }^{\circledR}$.

like circles, rectangles, or squares. Complex structures such as NCAs or CMP thus have to be built from combinations of these simple shapes in a time-consuming process.

The EFA represents a new, alternative, fast and simple scanning method. With this algorithm, it is possible to scan any arbitrary or complex pattern with minimal artefacts, requiring merely a 1-bit bitmap (BMP) file as input. EFA drives the beam in a path that is always parallel to the outline of the NCA pattern (Fig. 1). We are currently implementing an electrostatic scanning system which should further alleviate the hysteresis problems and reduce the exposure time.

\section{Results and discussion}

\subsection{PBW of three-dimensional NCAs devices}

Three-dimensional NCAs devices were fabricated in SU-8 3000 photoresist of $40 \mu \mathrm{m}$ thickness. The array of 16 micro-wells was aligned over pre-defined gold electrodes sites (Fig. 2). The dimensions of the micro-wells were about $20 \times 20 \mu \mathrm{m}$ wide and $40 \mu \mathrm{m}$ high. The micro-wells were connected by buried microchannels with dimension of $5 \mu \mathrm{m}$ wide. Gold electrodes are used to provide

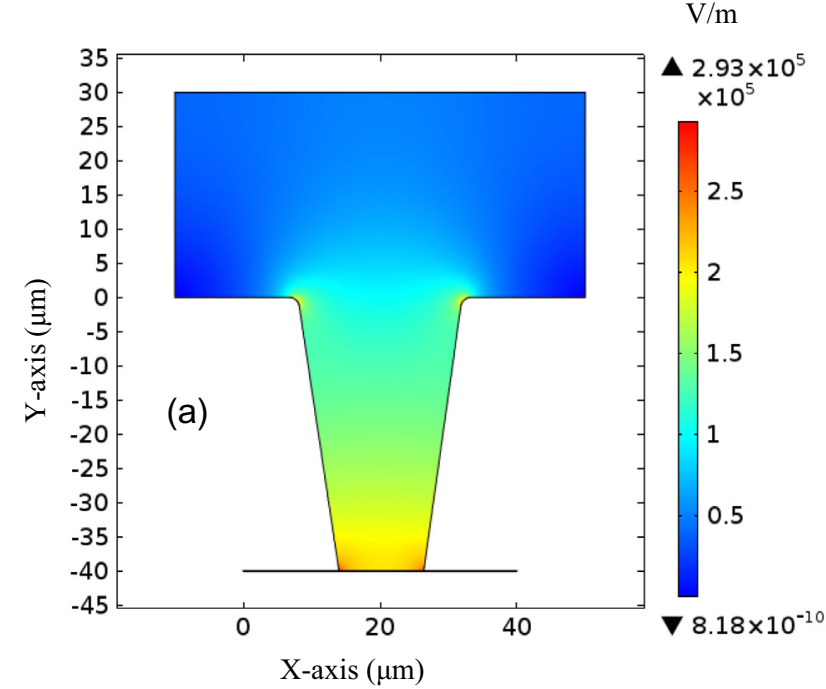

$\mathrm{V} / \mathrm{m}$

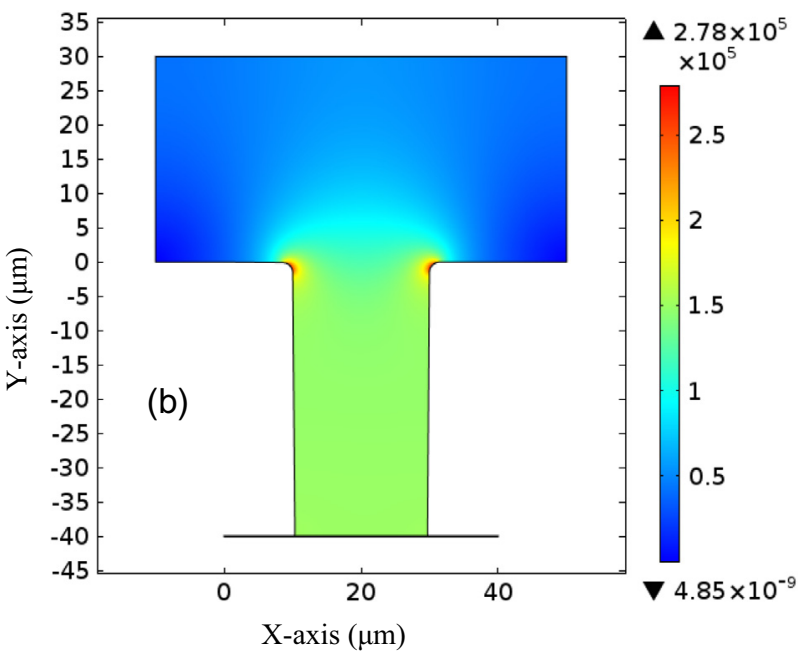

Fig. 6. Modelling of electric field distribution in DEP process where (a) is a microwell fabricated by UV-lithography and (b) is a micro-well pattern by PBW. UV exposure regime: wavelength $365 \mathrm{~nm}$, dose $150 \mathrm{~mJ} / \mathrm{cm}^{2}$, the SU-8 sample was aligned with the mask (JD Photo Tools, Oldham, UK) in contact mode using mask aligner (Ultra $\mu$ Line 7000 High Resolution Mask Aligner, Quintel Corporation, USA).

an electric field to exert a dielectrophoresis (DEP) force [24] on the cells to be localised inside the micro-wells. These electrodes also serve as probes to sense the action potential produced as a consequence of connection between neighbouring neurons [13].

The straightness of the wall and surface rms roughness in the NCAs channels was measured using Image software [25] and found to be about $89.5^{\circ}$ and $2.5 \mathrm{~nm}$ respectively (Fig. 3a and b). The buried channels in NCAs structure (Fig. 4) provide a link and guide for neurite growth in between single neurons that are isolated in the micro-wells. One of the major threats for cells at the micro-level is fast evaporation of the biological medium. Various techniques have been suggested to resolve this issue e.g., capping using a thermal bonding [12] or gluing of a covering layer [26]. However, these procedures often lead to a reduced functionality of the microfluidic device due to e.g., clogging of the channel or change of the channel's geometry.

By using PBW technique, the enclosed channel was fabricated in a single layer of resist in the course of two subsequent exposures. The numerical simulation of the fluid flow in this geometry shows 


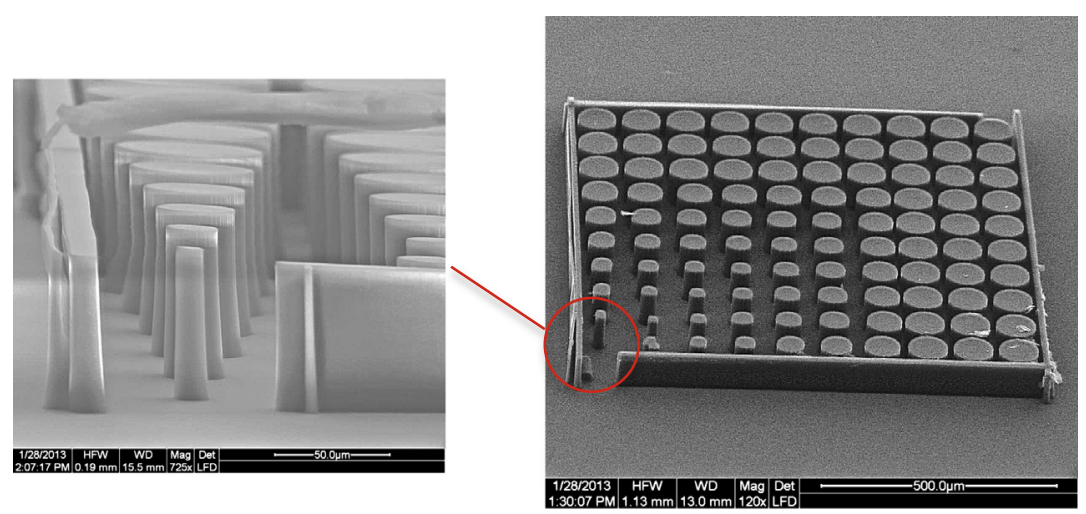

Fig. 7. SEM image of a prototype of CMP included a magnified tilted image of the pillars (55 $\mu \mathrm{m}$ high) dedicated for fluid flow tuning in CMP.

Time $=0.0012 \mathrm{~s}$

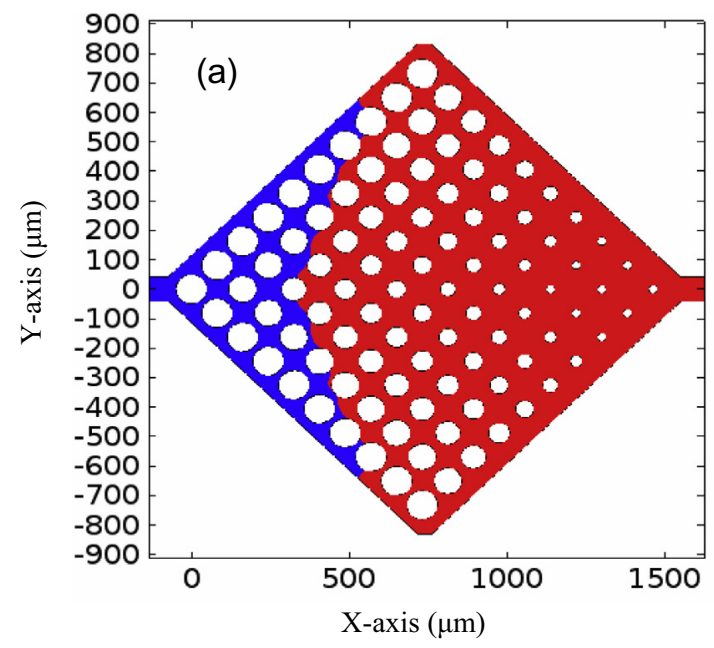

Time $=0.012 \mathrm{~s}$

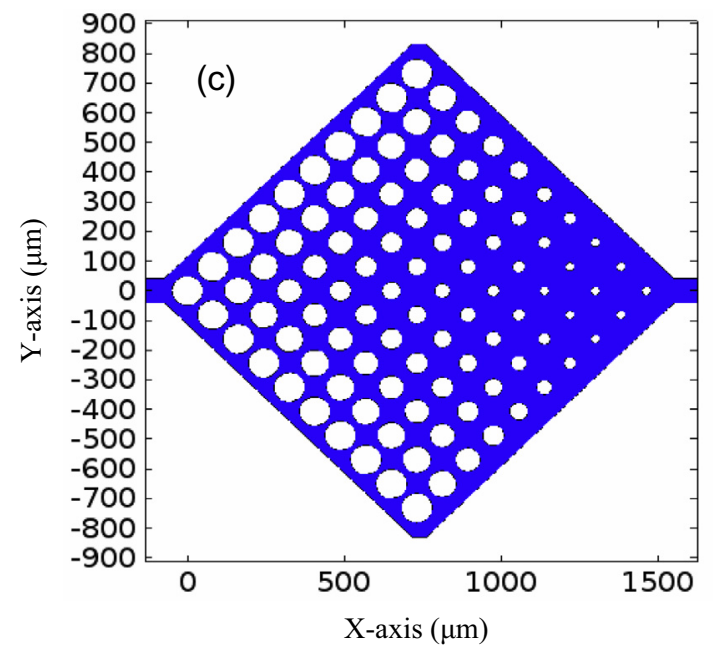

Time $=0.005 \mathrm{~s}$
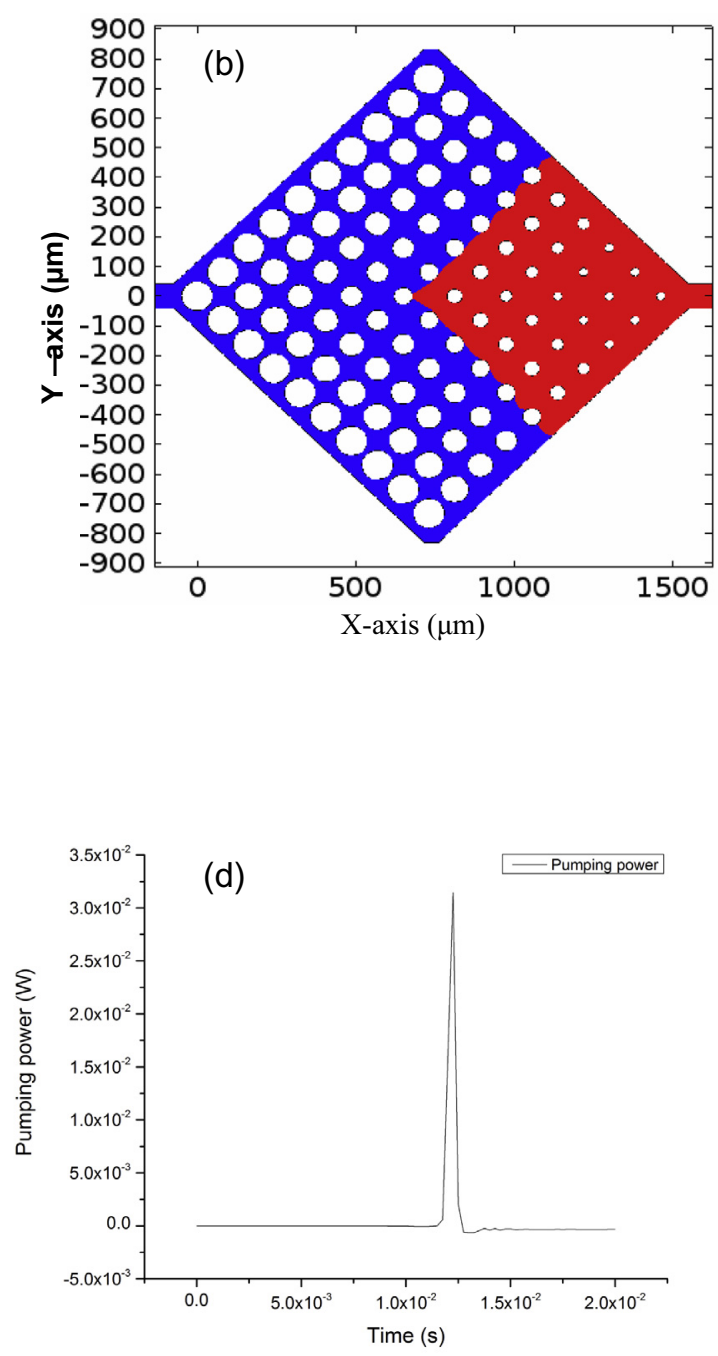

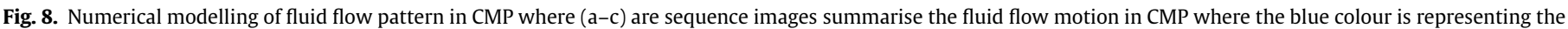

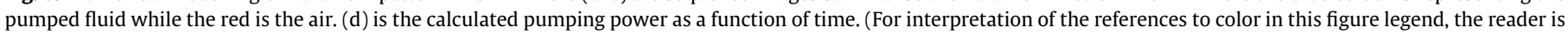
referred to the web version of this article.)

that the fluid velocity profile was uniform along the microchannel (Fig. 5). This parabolic profile of fluid flow in the rectangular chan- nel is critical to guarantee uniform distribution of the chemicals in a biological analysis. 

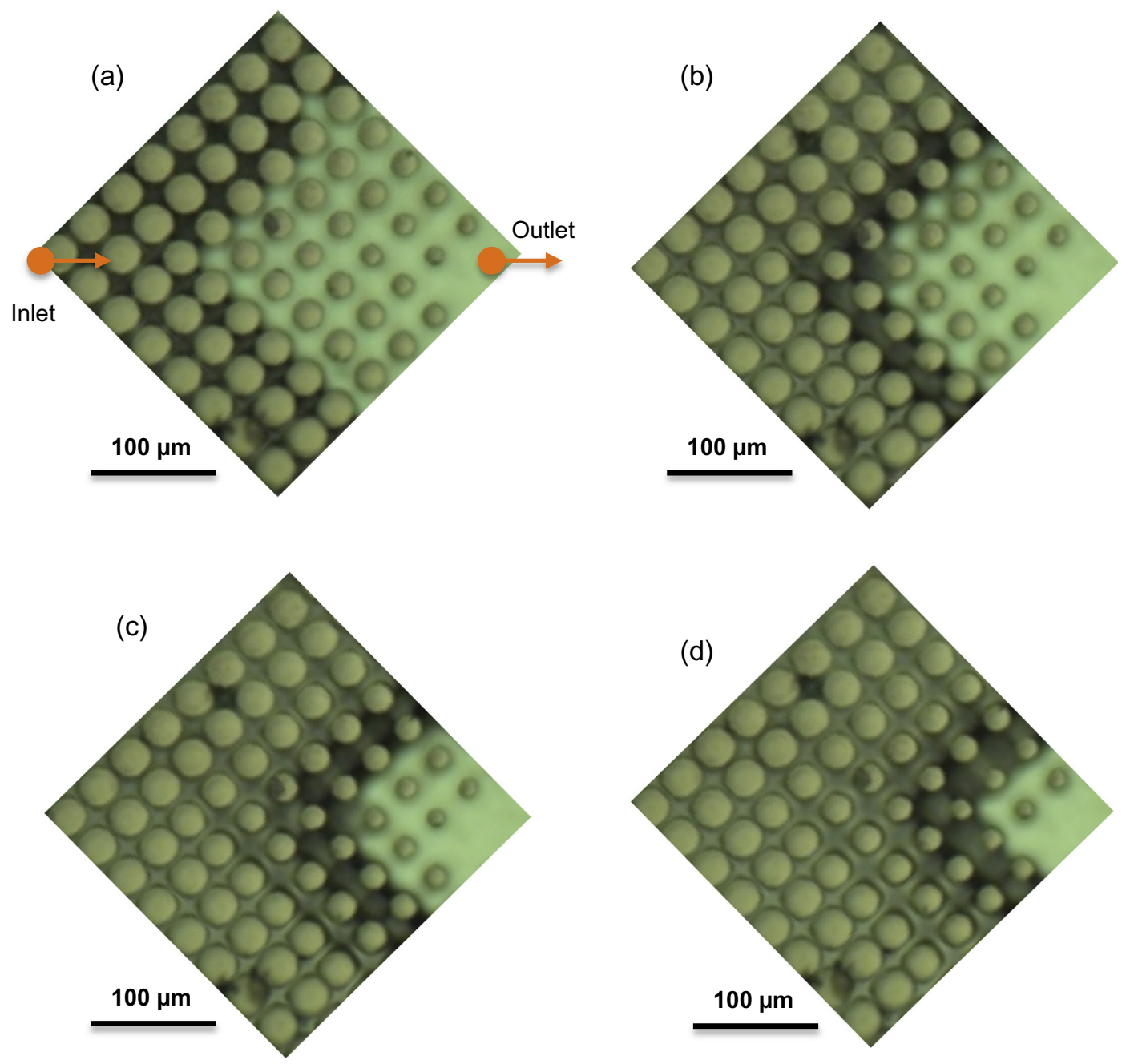

Fig. 9. Time-lapse of optical micrographs illustrating sequences of the fluid motion in CMP microfluidic fabricated by PBW.

The micro-wells fabricated by UV-lithography feature the negative slope walls that can create areas with the high electric field regions at the bottom edges of the micro-well (Fig. 6a) [13]. This in turn can cause an excessive cell death during dielectrophoresis (DEP) cell positioning, due to membrane rupture resulting from electroporation [27]. Contrary, the electric field gradient in the micro-well patterned by PBW is distributed evenly (Fig. 6b) as it was determined using analysis with COMSOL Multiphysics ${ }^{\circledR}$. The more uniform distribution of the electric field in proximity to the electrode site would expectedly contribute to a reduction in cell death rate.

\subsection{Capillary micro-pump (CMP)}

Capillary-based microfluidic systems are used in many bio-analytical applications to provide transport of the molecules and cells or to precisely control a delivery of reagents and drugs. A passive pump system with no moving parts is considered to be a preferred choice for these applications particularly for single-use devices $[28,29]$. The pumping efficiency of these devices can be controlled by the shapes and size of microstructures in the capillary pumps $[30,31]$. Here we present a prototype of a polymer capillary micropump (CMP) fabricated using PBW (Fig. 7). CMP consists of a set of cylindrical micropillars that are $55 \mu \mathrm{m}$ in height and $5-36 \mu \mathrm{m}$ in diameter. This arrangement creates different sizes of microchannels, which tunes the pressure throughout the CMP. Fluid flow rate is a function of pressure and fluid resistance. These two factors in turn are functions of the microchannel geometry and surface tension [32].

The process of fluid propagation and pumping in the CMP was modelled numerically using COMSOL Multiphysics ${ }^{\circledR}$. In Fig. 8(a-c) shows the fluid propagating from the inlet to the outlet forming L-shaped front filling sequentially each row of the micropillars. This causes a delay in filling each subsequent row, which in turn reduces the chance of air being trapped in between cylindrical pillars. According to the numerical calculation, this particular CMP can deliver a power of about $3.2 \times 10^{-2} \mathrm{~W}$, Fig. $8 \mathrm{~d}$. The pumping power can be expressed as follows:

$P=\Delta p \cdot Q$

where $\Delta p$ and $Q$ are the pressure drop across the CMP, and the fluid flow rate respectively. Besides being effective in pumping fluid, cylindrical pillars are also easy to design and fabricate.

Fluid flow in CMP was observed experimentally and recorded as time series of optical micrographs (Fig. 9). The liquid spreads along the edges of the pump and is then drawn to the next space between the micropillars rows. The filling front moves consistently along the main axis of the pump in good agreement with the numerical simulations. The surface of CMP was treated using Oxygen plasma (Plasma Asher Emitech K1050X) at a power of $75 \%$ for $5 \mathrm{~min}$. As a result, surface wettability increased by reducing the fluid contact angle [33]. 


\section{Conclusions}

Proton beam writing technique was used on SU-8 photoresist to fabricate three-dimensional (3D) microstructures of Neuron Cell Array for single-neuron study and capillary micropump as selfpowered microfluidics. The sidewalls of the 3D-NCAs had a great degree of straightness and a smooth surface. Primary results of computer simulations indicated that these structural improvements have the potential to provide a safe and efficient microenvironment for creating a neural network. The new scanning method (EFA) used in the present work enabled us to improve the quality of NCAs and CMP structures and provided more flexibility in designing the complex structures.

\section{Acknowledgement}

This work was sponsored by King Abdulaziz City for Science and Technology (KACST), Saudi Arabia.

\section{References}

[1] K. Ziolkowska, R. Kwapiszewski, Z. Brzozka, New J. Chem. 35 (2011) 979-990.

[2] I. Papautsky, B.K. Gale, S. Mohanty, T.A. Ameel, A.B. Frazier, Microfluid. Devices Syst. II 3877 (1999) 147-158.

[3] H.A. Stone, A.D. Stroock, A. Ajdari, Annu. Rev. Fluid Mech. 36 (2004) 381-411.

[4] T. Bayraktar, S.B. Pidugu, Int. J. Heat Mass Trans. 49 (2006) 815-824.

[5] F.O. Morin, Y. Takamura, E. Tamiya, J. Biosci. Bioeng. 100 (2005) 131-143.

[6] P.J. Hung, P.J. Lee, P. Sabounchi, N. Aghdam, R. Lin, L.P. Lee, Lab Chip 5 (2005) 44-48.

[7] A.N. Broers, Philos. Trans. R. Soc. A 353 (1995) 291-311.

[8] F. Cerrina, Mater. Res. Soc. Symp. Proc. 380 (1995) 173-177.

[9] L.R. Harriott, Proc. IEEE 89 (2001) 366-374.

[10] M. Maenhoudt, S. Verhaegen, K. Ronse, P. Zandbergen, E. Muzio, Proc. Soc Photo-Opt. Ins. 4000 (2000) 373-387.
[11] A.A. Bettiol, E.J. Teo, C.N.B. Udalagama, S.V. Rao, J.A. van Kan, P.G. Shao, F. Watt, Mems Moems Micromachining 6186 (2006) F1860.

[12] K.A. Mahabadi, I. Rodriguez, S.C. Haur, J.A. van Kan, A.A. Bettiol, F. Watt, J. Micromech. Microeng. 16 (2006) 1170-1180.

[13] F.T. Jaber, F.H. Labeed, M.P. Hughes, J. Neurosci. Methods 182 (2009) 225-235.

[14] F. Watt, M.B.H. Breese, A.A. Bettiol, J.A. van Kan, Mater. Today 10 (2007) 20-29.

[15] F. Zhang, F. Sun, J.A. van Kan, P.G. Shao, Z. Zheng, R.W. Ge, F. Watt, Nucl Instrum. Methods Phys. Res., Sect. B 231 (2005) 413-418.

[16] S. Homhuan, B. Zhang, F.S. Sheu, A.A. Bettiol, F. Watt, Biomed. Microdevices 14 (2012) 533-540.

[17] W. Larisch, T. Koal, R. Werner, M. Hohlweg, T. Reinert, T. Butz, Nucl. Instrum. Methods Phys, Res., Sect. B 269 (2011) 2444-2447.

[18] J.F. Ziegler, M.D. Ziegler, J.P. Biersack, Nucl. Instrum. Methods Phys. Res., Sect. B 268 (2010) 1818-1823.

[19] T. Osipowicz, J.A. van Kan, T.C. Sum, J.L. Sanchez, F. Watt, Nucl. Instrum. Methods Phys. Res., Sect. B 161 (2000) 83-89.

[20] M. Corp., SU-8 3000, MicroChem Corp., 2014.

[21] A.A. Bettiol, C.N.B. Udalagama, J.A. van Kan, F. Watt, Nucl. Instrum. Methods Phys. Res., Sect. B 231 (2005) 400-406.

[22] K.A. Stetson, Appl. Opt. 31 (1992) 5320-5325.

[23] K. Somkantha, N. Theera-Umpon, S. Auephanwiriyakul, IEEE Trans. Bio-Med. Eng. 58 (2011) 567-573.

[24] T.B. Jones, IEEE Eng. Med. Biol. 22 (2003) 33-42.

[25] W.S.R.a.J. Image, Image processing and analysis in JAVA, U.S. National Institutes of Health, Bethesda, MD, 1997-2006.

[26] K.T. Haraldsson, Fabrication of polymeric microfluidic devices via photocurable liquid monomers, Department of Chemical Engineering and Technology Fibre and Polymer Technology, KTH- Royal Institute of Technology, Stockholm, Sweden, 2005.

[27] T. Heida, J.B.M. Wagenaar, W.L.C. Rutten, E. Marani, IEEE Trans. Bio-Med. Eng. 49 (2002) 1195-1203.

[28] L. Gervais, E. Delamarche, Lab Chip 9 (2009) 3330-3337.

[29] T. Horiuchi, K. Hayashi, M. Seyama, S. Inoue, E. Tamechika, Sensors (Basel) 12 (2012) 14053-14067.

[30] E. Berthier, D.J. Beebe, Lab Chip 7 (2007) 1475-1478.

[31] M. Zimmermann, H. Schmid, P. Hunziker, E. Delamarche, Lab Chip 7 (2007) 119-125.

[32] D.J. Beebe, G.A. Mensing, G.M. Walker, Annu. Rev. Biomed. Eng. 4 (2002) 261 286.

[33] M. Corp., SU-8 Surface Modification - Functionalization, MicroChem Corp., 2014. 